\title{
Genesis
}

Manuscrits - Recherche - Invention

\section{L'illumination lointaine des tablettes d'argile. Le statut de « l'intertexte mésopotamien » dans la genèse de Vents de Saint-John Perse}

suivi de : Chronique d'une découverte génétique : entretien avec Christian Rivoire

\section{Esa Christine Hartmann}

\section{(2) OpenEdition}

\section{Journals}

Édition électronique

URL : http://journals.openedition.org/genesis/379

DOI : $10.4000 /$ genesis.379

ISSN : 2268-1590

Éditeur :

Presses universitaires de Paris Sorbonne (PUPS), Société internationale de génétique artistique littéraire et scientifique (SIGALES)

\section{Édition imprimée}

Date de publication : 10 octobre 2010

Pagination : 127-139

ISBN : $978-2-84050-711-6$

ISSN : $1167-5101$

Référence électronique

Esa Christine Hartmann, «L'illumination lointaine des tablettes d'argile. Le statut de « l'intertexte mésopotamien » dans la genèse de Vents de Saint-John Perse », Genesis [En ligne], 31 | 2010, mis en ligne le 22 février 2013, consulté le 02 mai 2019. URL : http://journals.openedition.org/genesis/379 ; DOI : 10.4000/genesis.379

Ce document a été généré automatiquement le 2 mai 2019.

Tous droits réservés 


\section{L'illumination lointaine des} tablettes d'argile. Le statut de « l'intertexte mésopotamien » dans la genèse de Vents de Saint-John

\section{Perse}

suivi de : Chronique d'une découverte génétique : entretien avec Christian Rivoire

\section{Esa Christine Hartmann}

" Et les voici, vocables assujettis au même enchaînement, pour l'exercice au loin d'une divination nouvelle ${ }^{1} . . . »$ Rares sont les découvertes intertextuelles qui permettent d'adopter une direction radicalement nouvelle dans l'interprétation d'une œuvre. Rares sont les trouvailles «archéologiques » qui dépoussièrent des documents longuement enfouis dans la nuit des archives, et réorientent la critique de façon inédite et inespérée. Rares sont aussi les révélations génétiques qui mettent en lumière les zones d'ombre d'une démarche créatrice, et nous approchent un tant soit peu de ce royaume convoité qu'est l'univers imaginaire d'un auteur. Nous sommes donc d'autant plus heureuse de présenter ici une découverte assez considérable: grâce aux fructueuses recherches entreprises par Christian Rivoire dans les archives de la Fondation Saint-John Perse d'Aixen-Provence, un élément primordial vient d'être ajouté au dossier génétique du poème Vents, qui fut composé par le poète au cours de son exil américain et publié en 1946 à Paris'.

2 Car énigmatique, la naissance de ce poème le fut toujours, par l'insuffisance de ses trois états manuscrits qui reflètent un stade très avancé dans la composition du poème, et qui ne sont précédés par aucun brouillon conservé ${ }^{3}$. Cependant, après de patientes et persévérantes investigations, de nombreuses sources intertextuelles ont pu être identifiées jusqu'à maintenant ${ }^{4}$, permettant d'expliquer l'origine d'un grand nombre d'images, d'idées et de tournures rhétoriques de Vents. Parmi ces sources intertextuelles 
figurent avant tout les livres appartenant à la bibliothèque personnelle de Saint-John Perse $^{5}$, livres à partir desquels les critiques ont découvert une véritable stratégie du collage et de l'emprunt ${ }^{6}$.

3 Ce sont principalement les ouvrages d'histoire et d'anthropologie, étudiant des textes sacrés comme le Dict de Padma ${ }^{7}$ et s'intéressant aux différentes formes de communication avec le divin dans les civilisations anciennes et les sociétés primitives, tel La Pensée de l'Asie et l'astrobiologie de René Berthelot ${ }^{8}$, qui participent activement à la conception de l'univers imaginaire de Vents, où foisonnent les rites d'initiation et de divination'. Or toutes ces informations intertextuelles ne parvinrent pas à combler l'incertitude exégétique provoquée par cette carence avant-textuelle, par ce chaînon manquant dans la genèse du poème.

4 C'est, de fait, un livre in absentia qui permit d'illuminer ce mystère, en apportant les éclairages nouveaux longtemps désirés, et dont l'infime trace nous est parvenue sous la forme d'un manuscrit de notes de lecture, que Saint-John Perse avait prises à partir d'un livre consulté. Il s'agit d'une publication innovante de l'époque en matière d'anthropologie, l'ouvrage d'histoire antique, La Divination chez les Assyriens et les Babyloniens, du D $\mathrm{D}^{\mathrm{r}}$ Georges Contenau (1877-1964), publié en 1940 chez Payot.

Ces notes de lecture à l'encre bleu marine, enfouies au fond d'un dossier longtemps inexploré, sont référencées sous la cote MS N 59 (voir fig. 1). Elles forment un manuscrit complet de dix-sept pages (format $21 \times 13,5 \mathrm{~cm}$ ) à l'écriture petite et aux nombreuses abréviations, couvrant la totalité du livre de G.Contenau, dont Saint-John Perse a fidèlement recopié des passages entiers ${ }^{10}$. Alors que le livre lui-même est introuvable au sein de la bibliothèque personnelle conservée à la Fondation Saint-John Perse, il s'avère d'autant plus présent dans la genèse de Vents. Car, contrairement à la pratique du collage, ponctuelle et secondaire, nous sommes ici en présence d'une technique d'emprunt plus complexe qui s'avère être un trait d'écriture majeur à la source même du poème ${ }^{11}$.

6 Ainsi la présente étude se proposera-t-elle d'analyser la fonction et le développement de cet étonnant « intertexte mésopotamien » à l'intérieur du processus d'écriture persien. Peut-être nous permettra-t-elle aussi de saisir cette étincelle poétique si fuyante qui, surgissant des tablettes d'argile sous le regard créateur de Saint-John Perse, enfantera les images du poème. Et c'est sans doute ici que nous assisterons à l'imperceptible transformation des notes de lecture en un atelier d'écriture, où naissent un style et une poétique, qui porteront l'écho lointain des formules magiques sumériennes.

7 Nous définissons l'emprunt comme une unité linguistique (dont le signifiant et/ou le signifié peuvent être exploités par le poète) et discursive (aux variables syntaxiques et stylistiques), incarnant à la fois un signe et un microrécit. Afin de retracer l'évolution génétique de cette unité intertextuelle, le plan de notre étude suivra le voyage de l'emprunt à travers les différentes étapes scripturales, jusqu'à son éclosion finale dans le texte poétique.

\section{L'émergence de l'emprunt dans les notes de lecture}

8 Témoignant d'une lecture à la fois scientifique et créatrice, les notes manuscrites prises à partir du livre de G. Contenau représentent la fondation documentaire du poème, ce référent livresque qui constitue une des sources premières de son inspiration, fournissant des images clés à partir desquelles vont proliférer les développements thématiques, 
foisonner les réseaux métaphoriques. Mais elles contiennent aussi déjà les traces d'une première lecture critique de la part du poète : en dehors des soulignements de passages particulièrement évocateurs que nous retrouvons, pour la plupart, réinvestis dans le poème, les signes d'annotations de Saint-John Perse - ronds, croix, traits verticaux envahissent l'espace marginal des feuillets. Dans les interlignes, en revanche, se dessinent les premières ébauches de variantes, témoignant d'une réelle écriture poétique. Ces réécritures ouvrent l'espace virtuel de l'invention, et convertissent les notes documentaires en un premier état manuscrit du poème naissant. Document liminaire donc, qui, sous la plume du poète autant que sous le regard du chercheur, incarne et «met en écriture» le seuil, souvent insaisissable, entre le travail de documentation exogénétique et l'avant-texte endogénétique, l'écriture poétique à proprement parler. Lors de ce passage de frontières avant-textuelles, allant de la simple prise de notes, du simple recopiage à l'élaboration des premiers paradigmes de variantes où s'aventure l'esprit créateur dans les possibles d'une image qu'il fait naître à partir d'un texte donné, en passant par le soulignement marquant la prise de possession du discours étranger, le poète s'approprie la source documentaire, l'investit de sa force imaginaire, l'épouse sous le trait de sa plume, pour la faire sienne pour toujours. Cette appropriation est, comme nous le verrons, double, thématique et stylistique, affectant pour ainsi dire le fond et la forme du poème à naître.

9 Si les notes documentaires converties en un véritable état manuscrit (qu'on pourrait peut-être appeler Ve 0) changent ainsi de statut génétique, les références intertextuelles ou emprunts que nous rencontrons dans le poème, apparaissent désormais comme des échos intratextuels dont les avatars voyagent d'état en état. Une telle appropriation de la source documentaire sous la plume du poète semble d'autant plus facile qu'elle se fonde sur une même loi d'analogie, régissant à la fois la pratique de divination (sujet du livre d'anthropologie de Georges Contenau dont se nourrit la pensée persienne), et le fonctionnement même du langage poétique persien - analogie qui associe pour toujours la mantique mésopotamienne à cette "mantique du poème » dont parle Vents. Georges Contenau évoque en effet un "monde sumérien profondément religieux où tout peut faire signe pour connaître la volonté divine, d'autant que le raisonnement est volontiers analogique $^{12}$ ». Analogie sur laquelle s'appuie aussi la poétique persienne, comme le déclare le fameux Discours de Stockholm, prononcé lors de la remise du prix Nobel en 1960 : «Par la pensée analogique et symbolique, par l'illumination lointaine de l'image médiatrice, et par le jeu de ses correspondances, sur mille chaînes de réaction et d'associations étrangères, par la grâce enfin d'un langage où se transmet le mouvement même de l'Être, le poète s'investit d'une surréalité qui ne peut être celle de la science ${ }^{13}$. »

Curieusement, la loi d'analogie qui préside à l'interprétation des augures guide aussi la lecture des images poétiques: dans Vents, le «chaman » est un double du poète. Face aux signes divins visibles dans la nature, l'exégèse divinatoire et l'écriture poétique, suivant la même pratique herméneutique, se confondent dans la poésie persienne. Par conséquent, l'emprunt se développe souvent en leitmotiv, ce qui augmente son pouvoir magique, que Saint-John Perse puise justement dans les tablettes de divination contenant les formules rituelles des enchanteurs anciens :

G. Contenau, p. 105 [enquête de l'enchanteur auprès de son malade] :

A-t-il enlevé des clôtures, des bornes?

Saint-John Perse, Vents, I, 6, versets 42 et 53 :

Enlèvement de clôtures, de bornes 
11 La plume du poète trace la "voie mantique » dont parle Georges Contenau, car elle matérialise les rêves divinatoires passant par la «porte de corne »: «Et il y avait cette année-là, à vos portes de corne, tout ce parfum poignant de bêtes lourdes, mufle bas, sur les divinations errantes de la terre la rumeur croissante des conques souterraines » (Vents, II, 2). Comparons ce verset au texte de Contenau : « La voie mantique la plus communément attestée dans Homère, est l'observation des actions et des cris des oiseaux, principalement de l'aigle envoyé par Zeus, Apollon ou Athênè. Une part importante est accordée aux rêves, selon qu'ils sont venus par la porte d'ivoire ou par la porte de corne ( Odyssée, IXI, 560)» (op.cit., p. $36^{14}$. Notons que la référence du texte homérique dans le texte persien est de seconde main ici).

12 À l'instar du travail de composition effectué à partir du dictionnaire analogique ${ }^{15}$, les notes de lecture, où apparaît une véritable poétique de la liste, représentent l'équivalent d'un « syllabaire »: «Et comme un songe prénuptial où l'homme encore tient son rang, à la lisière d'un autre âge, interprétant la feuille noire et les arborescences du silence dans de plus vastes syllabaires » (Vents, II, 1, 12) ${ }^{16}$. En effet, les notations de Saint-John Perse, précédées chacune par un tiret en début de ligne marquant une unité textuelle autonome, exposent, bien qu'elles suivent la progression linéaire du texte de source, une structure verticale, paradigmatique : elles fonctionnent comme une immense palette, un répertoire à idées, images et rythmes, un catalogue abondant où le poète choisit l'emprunt à insérer dans le mouvement syntagmatique du poème naissant, où il s'anime d'une vie nouvelle.

Quelle est cependant la force d'inspiration de l'élément intertextuel, sa poéticité ? Parmi les annotations de Saint-John Perse, nous retrouvons certes les images phares de son univers imaginaire: ses animaux de prédilection, tels le cheval ${ }^{17}$ et l'aigle ${ }^{18}$, symboles chamaniques incarnant un pouvoir sacré et initiatique, auxquels s'ajoutent les animaux divinatoires des augures, le serpent ${ }^{19}$, la chouette ${ }^{20}$ et les insectes ${ }^{21}$. Ensuite, en accord avec l'abondance des expressions métatextuelles dans ses œuvres, ce sont surtout les termes appartenant au champ lexical de l'écrit et des formes poétiques qui intéressent Saint-John Perse ${ }^{22}$ : « syllabaire » (Vents II, 1, 12), « allitérations », " jeux d'écriture » ( Vents IV , 5, 9), « calembours », « dossier » (IV, 5, 9), « copies » (III, 6, 11), « originaux » (III, 6, 11), « tablettes », « livres d'argile » (I, 4, 3 et I, 5, 16) « parchemin », « signes » (I, 5, 25 et I, 6, 32), " roseau », " calame ", « scribe ", « texte ", " bibliothèque ", " incantation », " textes rituels ", " amulettes », « inscriptions sur pierre », " rédaction de présages », «idéogrammes ». En dernier lieu, les différents avatars du Poète dans Vents contiennent également de nombreux emprunts : «l'officiant » (III, 3, 18 et III, 4, 18), le « devin », le « prêtre » (I, 4, 4), le « voyant » (III, 6, 29), « l'écoutant » (ibid.), « l'enchanteur » (I, 5, 25 et I, 6, 10 et III, 4, 15), "l'incantateur », le « chantre », « l'haruspice », le « médecin » et le «juge ». Source d'inspiration, vivier documentaire et linguistique où la plume du poète puise avec avidité, la force et la saveur poétiques des images rencontrées lors de la lecture documentaire stimulent ainsi l'imagination créatrice du poète et conditionnent la naissance des réseaux métaphoriques dans Vents.

\section{La transformation de l'emprunt : procédés d'écriture}

14 À la lumière du texte de Georges Contenau, truffé de définitions scientifiques, l'invention scripturale de Vents se transforme en un véritable rite divinatoire, que Saint-John Perse nomma la «mantique du poème » : «Reconnaissant pour excellente cette mantique du 
poème, et tout ce qu'un homme entend aux approches du soir » (Vents, I, 2). Ce verset contient trois échos combinés du texte de Contenau, qui définit ainsi le terme de «mantique ${ }^{23} »:$ " La voie mantique la plus communément attestée dans Homère, est l'observation des actions et des cris des oiseaux, principalement de l'aigle envoyé par Zeus, Apollon ou Athênè » (p. 36) ; "Présages tirés [...] de ce qu'entend un homme approchant un dieu [...]» (p. 102); «Ces prophètes parlent dans l'enthousiasme et Mursil II qui eut assez souvent recours à la divination envisage de s'adresser à cette mantique particulière en raison sans doute de son excellence » (p. 129).

15 Le génie combinatoire du poète dévoile ici les différentes vertus de l'emprunt: les déclinaisons sémantiques (définitions d'un terme technique, tel que «mantique ») aident à élaborer les branches thématiques du poème (dans notre cas, la mission du Poète ${ }^{24}-$ devin - Officiant $t^{25}$, la manifestation des différents signes et présages divins); les déclinaisons paradigmatiques, possibles de l'invention, enrichissent le trésor sémique du texte poétique (approche - présage - mantique/excellence - enthousiasme) ; la procréation syntagmatique, oscillant entre répétition et variation, conduit à un mouvement de dissémination textuelle (célébré, au niveau fictionnel, dans l'action des vents), qui stimule l'engendrement scriptural, comme dans l'emprunt suivant :

« [...] Eâ, dieu de l'abîme, ton bâillement n'est pas plus vaste » (Vents, I, 5).

« [...] Eâ, dieu de l'abîme, les tentations du doute seraient promptes » (Vents, I, 7).

"Ea dieu de l'abîme où est contenu tout savoir » (G. Contenau, p. 79).

16 Tandis que la procréation syntagmatique de l'emprunt transforme celui-ci en véritable leitmotiv, ponctuant la scansion litanique d'une invocation rituelle, sa transfiguration stylistique, qui consiste ici dans le simple ajout de deux virgules, change son statut sémantique: nous passons d'une apposition à valeur explicative, d'une qualification scientifique dans le texte de Contenau, à une apostrophe servant à invoquer la divinité allégorisée et son règne abyssal (Eâ incarne désormais un personnage intradiégétique, prenant part à l'action et au dialogue poétiques). La présence de l'accent circonflexe ( $\mathrm{E}$ $\hat{a} »)$ dans le texte persien représente d'ailleurs un avatar poétique du vocatif latin, marquant l'apostrophe, l'invocation lyrique.

La métamorphose de la source intertextuelle sous la plume de Saint-John Perse suit donc des mécanismes scripturaux certains ${ }^{26}$, engendrant une véritable stylistique transformative, que le poète lui-même appelle peut-être "transpositions, stylisations et créations ${ }^{27}$ ». Parmi ces constantes d'écriture régissant l'insertion de l'emprunt dans le texte poétique naissant, le phénomène d'inversion s'avère le plus récurrent. Citons d'abord Georges Contenau : «Les stations (manzazu) sont ce que les Grecs ont appelé l'hyposoma, endroit où les planètes ont leur "exaltation". [...] Les trois étoiles de chaque mois se succèdent dans leur coucher héliaque " (p. 312). «Le fait qu'Ishtar [" déesse de fertilité et de fécondité, assimilée à Vénus »], selon la saison de l'année, était astre du soir ou astre du matin, donnait une grande variété à ses présages : son coucher héliaque était, comme pour la lune, un enlèvement ${ }^{28} »$ (p. 323). Et Saint-John Perse répond : « Les trois étoiles mensuelles se succédant encore dans leur coucher héliaque et la révolution des hommes s'aggravent en ce point de l'année où les planètes ont leur exaltation » (Vents, IV, 4).

Le réinvestissement des syntagmes empruntés obéit ici à un mouvement chiasmique, que Loïc Céry a appelé la rhétorique " d'inversion ${ }^{29}$ ». Dans notre exemple, nous lisons dans le texte de G. Contenau : A1. «planètes [...] exaltation »-B1. « étoiles [...] héliaque », tandis que le texte persien commence par B2. «étoiles $[. .$.$] héliaque », suivi par A2. «planètes [. .$. 
] exaltation ». Dans une perspective synchronique, la réécriture de l'emprunt suit ainsi le paradigme $\mathrm{AB} / \mathrm{BA}$, ce qui correspond à la figure rhétorique du chiasme.

Affranchissant l'emprunt de son passé syntaxique en lui imprégnant un rythme nouveau, Saint-John Perse prend poétiquement possession du discours étranger, le fait sien en l'animant de son souffle poétique. Observons un autre exemple astrologique, où les références, à plusieurs pages d'intervalle, cohabitent chez Saint-John Perse dans un même verset. G. Contenau écrit: «une tablette du British Museum trouvée à Ninive s'exprime ainsi : "Mars à sa plus grande puissance devient splendide et reste ainsi plusieurs semaines successives [...] La visibilité de Mercure est proche. Il faut prévoir un grand éclat. [...] Ce qu'on voit maintenant, c'est Mercure dans la constellation du Capricorne" " (p. 310 sq.). Et voici, dans le poème de Saint-John Perse, le réinvestissement en forme chiasmique (A1. «Mars» B1. «Mercure» / B2. «Mercure » A2. «Mars ») : «Et la visibilité de Mercure est encore proche dans la constellation du Capricorne, et Mars peut-être à sa plus grande puissance se tient, splendide et vaste, sur la Beauce» (Vents, IV, 4). Le réarrangement des syntagmes crée une mélodie et un rythme nouveaux, transformant le texte antique en un verset poétique.

La narrativisation représente un autre phénomène scriptural qui accompagne l'intégration de l'emprunt dans le mouvement diégétique du poème, tout en l'imprégnant d'une historicité. Dans ce cas, l'emprunt est accompagné d'une indication temporelle (" jadis ${ }^{30} »$, "d'antan $\left.{ }^{31} »\right)$, qui semble non seulement renvoyer, au niveau sémantique, à l'antique civilisation mésopotamienne, mais aussi à une antériorité textuelle (ou plutôt inter textuelle), et incarner ainsi une allusion oblique au texte de source, une analepse vers le processus de création, remontant à l'origine exogénétique de l'emprunt. Surgit ainsi un discours double, traduisant à la fois un passé intradiégétique/fictionnel et extradiégétique/génétique.

21 La narrativisation d'une mention autonymique du texte de source, apparaissant dans la forme d'une citation des tablettes assyriennes marquée par des guillemets, représente un autre moyen de transfiguration de l'emprunt, en l'intégrant dans l'action fictionnelle du poème. Ainsi, dans le discours scientifique de Contenau, une expression en mention est analysée sémantiquement, sans qu'aucun personnage vienne soutenir l'action représentée. Elle figurera en usage dans le verset persien, remplissant une fonction dans la diégèse du poème :

Vents, III, 5, 15 :

Ou comme l'Initié, aux fêtes closes de la mi-nuit, qui entend tout à coup céder le haut vantail de cèdre à la ruée du vent - et toutes torches renversées, dans la dispersion des tables rituelles s'aventurent ses pas, et le filet du dieu d'en bas s'est abattu sur lui, et de toutes parts l'aile multiple de l'erreur, s'affolant comme un sphex, lui démêle mieux sa voie.

Contenau, p. 68 :

[les prêtres] doivent prêter serments. Comme le remarque M. Dhorme, à propos de cette lettre, l'expression littérale est «ils entrent à l'intérieur des serments »; le serment est un filet dont on ne peut sortir. Une idée analogue est rendue par l'expression "que le filet du dieu s'abatte sur lui ", et par la stèle des Vautours, du Musée du Louvre, où les ennemis du dieu sont représentés en son pouvoir dans un filet.

Dans le verset de Vents, l'expression empruntée intègre le discours du Poète, comme le montre d'ailleurs le passage d'une formule de conjuration rituelle (au présent du subjonctif optatif) au discours narratif allégorique (au passé composé de l'indicatif), introduit par une comparaison. Elle appartient désormais à un univers fictionnel, 
représenté ici par les aventures nocturnes de l'Initié, aux prises avec le « dieu d'en bas ». L'emprunt se plie ainsi aux exigences rythmiques et diégétiques du poème naissant, en changeant sa disposition syntaxique, sa courbe mélodique, son statut énonciatif. Par la narrativisation de l'emprunt, la dynamique fictionnelle du texte poétique s'empare du référent intertextuel, en lui conférant une véritable fonction diégétique.

\section{La valeur herméneutique de l'intertexte mésopotamien}

C'est donc dans un livre d'anthropologie antique que Saint-John Perse puisa les ingrédients thématiques et textuels pour les transformer, par une véritable " alchimie du verbe ", en cet or poétique dont rayonne le poème Vents. Assurément, nous sommes ici en présence d'une découverte de grande envergure - ce ne sont pas moins de quatre-vingtdeux versets de ce long poème qui portent l'empreinte précise du texte de G. Contenau ${ }^{32}$ -, qui influe sur l'interprétation générale de l'œuvre, car il s'agit, comme le constate Loïc Céry, de « réorienter radicalement cette thématique divinatoire du pôle chamanique, provenant des Indiens d'Amérique, vers ce nouveau pôle mésopotamien, jusqu'alors inconnu en ce qui concerne Vents. ${ }^{33}$ Par conséquent, de nombreux passages enfantés par une inspiration toute différente, adoptent une signification nouvelle. À titre d'exemple, le verset que nous avons tenu pour le fruit d'une imagination érotique débridée sur le manuscrit Ve 1 (notamment l'image très directe de l'exhibition des " parties génitales ", qui semblait si bien illustrer le dynamisme d'Éros à l'origine de la puissance créatrice), censurés $^{34}$ par la suite, n'est en vérité que la transcription poétique de la célébration du taureau sacré :

G. Contenau, p. 193 :

De même lorsque Diodore nous décrit les fêtes qui suivent la reconnaissance du taureau sacré Apis : «Pendant les quarante jours indiqués, le taureau sacré n'est visible qu'aux femmes; elles se placent en face de lui et découvrent leurs parties génitales. »

Notes documentaires de Saint-John Perse :

$\mathrm{O}^{35} \mathrm{cf}$. Diodore a. v. fêtes q. suivent la reconnaiss. du taureau sacré Apis : «pend. les $40 \mathrm{j}$. indiqués, le taureau sacré n'est visible qu'aux f.; elles se placent en face de lui et découvrent leurs parties génitales ${ }^{36}$ ".

Manuscrit Ve 1, p. 6 :

Femme < odorante > / loisible au flair $\mathrm{du}<$ dieu > / ciel et à elle seule découvrant la perfection de son être/arôme/mettant à nu/vif ses parties génitales/l'intimité vivante de son être/corps

Texte définitif :

« Femme loisible au flair du Ciel et pour lui seul mettant à vif l'intimité vivante de son être... » (Vents, I, 5, O.C., p. 189).

La sensualité hiératique de l'image fascine Saint-John Perse, puisqu'elle semble évoquer l' archaïque dans toute sa saveur insolite, dans toute sa splendeur palpable. Le potentiel poétique incarné dans ce rite de divination transforme le référent lointain et sacré en un être nouveau, en une métaphore de la vivacité de l'Être. Le manuscrit Ve 1 démontre ainsi souvent, à travers ces variantes supprimées ultérieurement, "la filiation, ensuite gommée, avec le texte de Contenau ${ }^{37} »$. Dans notre cas, l'emprunt dans le texte persien représente une citation de citation (Contenau citant lui-même le texte de Diodore) ; cette citation au deuxième degré, désormais intradiégétique, intégrée à l'univers fictionnel de Vents, a perdu toute connotation autonymique : le « référent palimpseste », caché dans le 
texte de Saint-John Perse, est transfiguré sous l'influence de son nouveau contexte poétique.

Ailleurs, l'emprunt révèle une source livresque là où le critique avait cru voir une expérience réelle. Dans Vents, II, 4, Saint-John Perse écrit :

Les migrations de crabes sur la terre, l'écume aux lèvres et la clé haute, prennent par travers des vieilles Plantations côtières enclouées pour l'hiver comme des batteries de Fédéraux. Les blattes brunes sont dans les chambres de musique et la réserve à grain ; les serpents noirs lovés sur la fraîcheur des lins, aux buanderies de camphre et de cyprès.

Comment ne pas voir ici une réminiscence de l'exubérante faune exotique dont resplendissait l'univers antillais d'Éloges (1911), ce paradis d'enfance qui ne cesse de hanter l'imagination créatrice du poète? Les "vielles Plantations côtières", les « chambres de musique » et la « fraîcheur des lins » ne semblent-elles pas ressusciter ces éléments biographiques éclatants, traversant l'œuvre persienne jusqu'au " Grand âge » de Chronique (1960), où resurgissent le « linge des servantes », le « lit de l'aïeule » et, surtout, les instruments de musique, le "vieux gong de bronze », le «bois de luthier de l'épinette ou de la harpe $\mathrm{e}^{38}$ " peuplant les «chambres de musique» dans Vents? Et pourtant, le lecteur fait fausse route, car c'est à nouveau une tablette assyrienne qui représente la source véritable de ce cortège animalier, dont le texte persien incarne cette fois-ci une citation de troisième main (le texte assyrien est traduit par A. Boissier, cité par G. Contenau). Voici ce que dit l'ouvrage d'anthropologie antique ${ }^{39}$ :

Le vocabulaire de la faune assyrienne nous est imparfaitement connu [...] Pour les insectes, notamment, nous en sommes réduits aux conjectures; c'est donc sous toutes réserves que nous donnons le sens de blattes, qui paraît mieux convenir, comme le propose le traducteur, au texte suivant (A. Boissier, présages fournis par certains insectes). " Si des blattes brunes et noires sont vues dans la maison d'un homme [...] Si des blattes sont vues dans la réserve à blé [...] Si des blattes sont vues dans la réserve à sésame [...]» (p. 223), «Si un serpent est lové sur le lit de quelqu'un : les yeux de la femme de l'homme en question se tourneront (?) et elle vendra ses enfants pour de l'argent (p. 222).

Dans le texte poétique, palimpseste indéchiffrable, les éléments biographiques (la chose vécue) et les éléments intertextuels (la chose lue) sont inextricablement entrelacés; dans la conscience créatrice du poète, le texte assyrien fait revivre le souvenir antillais, déclenche le processus de remémoration. Par conséquent, le trait peut-être le plus éminent de la poésie persienne, l'inventaire du réel dans toute sa richesse insolite, s'animant sous le regard du poète illuminé (au sens rimbaldien), est le fruit d'un inventaire textuel, où transparait la force incongrue de l'archaïque.

Enfin, le référent intertextuel nous livre de précieuses informations pour éclaircir les images obscures du texte persien, comme dans ce verset: « De jeunes bêtes sous l'écume et d'hommes en armes s'ébrouant dans le torrent d'Arbelles» (Vents, IV, 5). Passage mystérieux, réunissant une image guerrière («hommes en armes", "bêtes sous l'écume ») à un nom énigmatique, "Arbelles ", que seule la déesse Ishtar dans le texte de G. Contenau peut mettre en lumière ${ }^{40}:$ «le culte d'Ishtar de la ville d'Arbèles, déesse de la guerre » (p. 128). Dans le poème, l'identité de la déesse est omise, seuls y figurent le lieu de son culte (dont l'orthographe est légèrement modifiée) et ses attributs guerriers. Sur le manuscrit des notes documentaires, en revanche, cette métaphore in absentia se transforme en une métaphore in praesentia. La source inspiratrice facilite ainsi la compréhension du passage, par une « illumination lointaine de l'image médiatrice ${ }^{41}$ ». 
Chez Saint-John Perse, l'emprunt donne naissance à deux modes de réécriture. Si l'emprunt porte sur le signifié du texte d'origine, il transporte une information documentaire, scientifique, que le poète intègre dans l'architecture sémantique du texte en devenir, introduisant ainsi « le réel dans le poème ${ }^{42}$ ». Dans ce cas, l'intertexte possède une valeur herméneutique, révélant la signification d'un terme obscur, comme dans le cas de la fameuse «plante Pî-lu » (Vents, III, 6), qui avait provoqué tant d'investigations érudites et que nous retrouvons enfin dans l'ouvrage de Contenau parmi les plantes hallucinogènes, "auxiliaires de la mantique ${ }^{43}$ » : «La Mandragore officinale de Linné est une solanée que les Assyriens ont connue sous le nom de pi(l)-lâu (en sumérien NAM-TARIRA), plante du dieu de la peste [...]. La mandragore a été dans l'antiquité et au moyen âge une plante éminemment magique, employée notamment comme analgésique ${ }^{44}$. »

Si, en revanche, l'emprunt porte sur le signifiant de la référence intertextuelle, sa matière sonore ouvre une dimension ludique, que l'imagination associative du poète exploite pour créer de nouvelles images à partir d'un jeu de mots. Examinons d'abord le texte persien : «Et que l'Aigle pêcheur, dans tout ce bel émoi, vienne à lâcher sa proie sur la piscine de vos filles, c'est démesure encore et mauvais goût dans la chronique du poète » (Vents, II, 4). Ce verset semble avoir emprunté le signifié d'une tablette mésopotamienne - « Si un oiseau (de proie) laisse tomber dans la maison de quelqu'un de la viande, un oiseau ou quelque chose d'autre qu'il ait pris, le possesseur de la maison vivra dans la splendeur ${ }^{45} \gg-$, mais il joue également avec le signifiant de la nomenclature scientifique utilisée par G. Contenau, car la « piscine de vos filles » n'a-t-elle pas été enfantée par « l'extipicine » (divination par les viscères) et «l'haruspicine » (divination par les entrailles) ${ }^{46}$ ?

Détachées de leur environnement livresque, les notes documentaires de Saint-John Perse s'animent sur le manuscrit d'une vie nouvelle; elles se convertissent en un premier état d'invention où peu à peu s'agencent les vers du poème naissant, où se combinent sons et images pour créer un monde nouveau. La divination incarne aussi une technique herméneutique, puisque le poète-devin (tout comme le lecteur) déchiffre les signes divins (ou langagiers) à la recherche d'un sens irrévélé. Une telle conception symboliste de la poésie adopte dans la démarche créatrice persienne une nouvelle signification : il s'agit d'interpréter la référence intertextuelle comme on lirait le vol d'un oiseau, pour deviner le sens poétique de cette matière première, le présage d'un verset à naître sous la plume du poète. Car, comme le dit si bien Loïc Céry, «l'essentiel est qu'au terme de cette transposition, l'art de la divination devient la "mantique du poème", appellation générique tant commentée où se vérifie la marque de Perse lui-même : il s'agit de placer la divination au centre de la démarche poétique ${ }^{47} »$. La mantique mésopotamienne ne représente donc pas seulement ce vivier fécond dont se nourrit le poème, mais aussi le mode de fonctionnement de la pensée créatrice et du langage poétique persiens. C'est grâce à elle que « le poète s'investit d'une surréalité qui ne peut être celle de la science ${ }^{48}$ » - « si d'argile se souvient l'homme ${ }^{49}$ ". 
Fig. 1 : Saint-John Perse, Notes de lecture

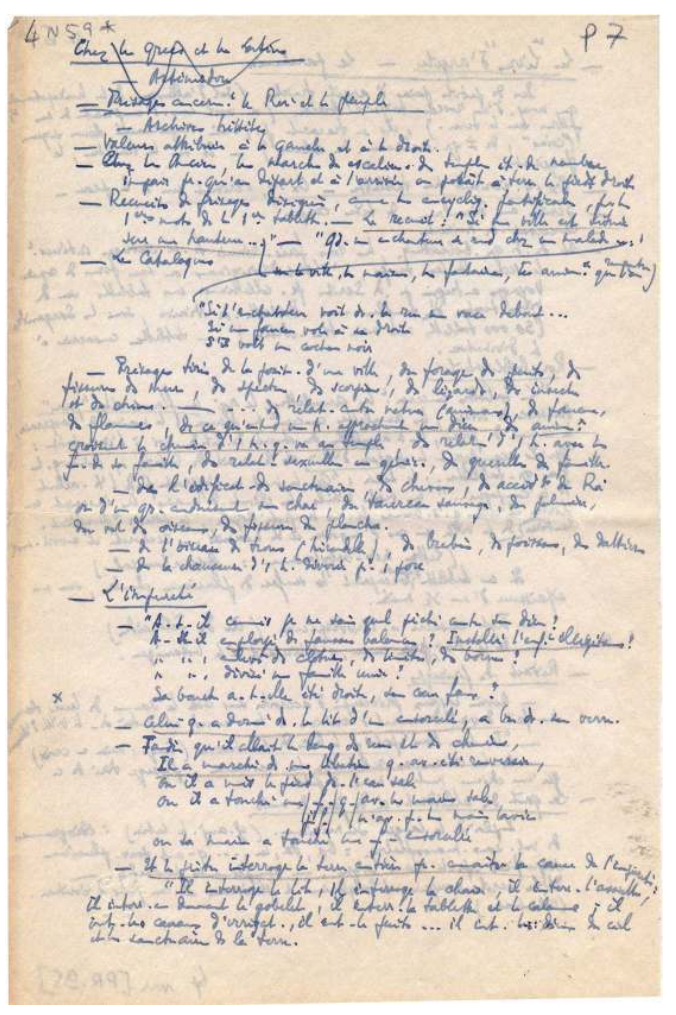

Ms N 59, p. 7

Fondation Saint-John Perse

\section{Chronique d'une découverte génétique : entretien avec Christian Rivoire}

Esa Christine Hartmann - Par votre étonnante découverte dans les archives de la Fondation Saint-John Perse d'Aix en Provence, vous avez éclairci une grande partie de la genèse de Vents, et conduit à une réorientation fondamentale de son interprétation. Quelles ont été les étapes herméneutiques qui vous ont conduit au livre de G. Contenau?

Christian Rivoire - Il y a quelques années, je visitais le département des Antiquités orientales au musée du Louvre. J'admirais ces signes cunéiformes sur les tablettes d'argile... En lisant le Code de Hammourabi, je crus saisir une certaine parenté de ton avec Saint-John Perse. Rentré chez moi, je consultai la liste complète des manuscrits référencés à la Fondation Saint-John Perse. En la parcourant rapidement, je découvris quelque chose sur les Babyloniens que je marquai d'une croix, en pensant qu'une piste se présentait peut-être de ce côté-là. Mais je l'oubliai par la suite, préférant chercher dans L'Épopée de Gilgamesh un éventuel ascendant babylonien sur la poésie persienne. Erreur méthodologique. Ce n'est pas dans les œuvres littéraires qu'on a le plus de chance de trouver une influence directe et forte sur Saint-John Perse...

Un jour que je travaillais à la Fondation Saint-John Perse (un vendredi treize !), je repris ce papier marqué de mes petites croix, que j'avais sous le coude depuis peut-être deux ans; je me décidai enfin à vérifier cette piste à propos des Assyriens. Je consultai la note documentaire manuscrite, et là... cadeau des dieux !... je trouve dix-sept petits feuillets, 
remplis de l'écriture fine de Saint-John Perse (avec ses abréviations habituelles), qui tout à la fois renvoient à un ouvrage de Georges Contenau et présentent une parenté saisissante avec le poème Vents! Je me demande de quoi il s'agit exactement : est-ce un état manuscrit du poème Vents antérieur à celui que nous appelons le manuscrit Ve 1 ? Mais alors, pourquoi cette référence à G. Contenau? Ou suis-je bien en présence de notes de lecture? Dans ce cas, l'influence de cet intertexte dans la genèse de Vents serait considérable.

Comme le livre en question n'est pas conservé dans la bibliothèque personnelle du poète à la Fondation, je me précipite sur Internet pour acquérir l'ouvrage. Heureusement, un exemplaire est encore en vente, je le commande - imaginez-vous l'attente pendant plus d'une semaine! Arrive le livre, je l'ouvre, et là, j'ai la confirmation de ce que je pressentais : ces notes de lecture reprennent littéralement les formulations de G. Contenau, il n'y a que très peu de modifications. Retournant enfin à ma lecture de Vents pour une mise en regard des deux textes, je liste, a minima, quatrevingt-deux versets de Vents où figurent un ou plusieurs emprunts à G. Contenau. Mais ce n'est pas tout...

E. C. H. - Faites-vous ici allusion à un autre hypotexte que vous avez découvert?

C.R. - En effet, j'ai continué à explorer les notes documentaires disponibles à la Fondation Saint-John Perse et j'ai mis à jour un autre corpus d'emprunts très important, à savoir certains travaux de l'ethnologue Alfred Métraux. Voici un exemple : dans la strophe IV du poème, le héros de Vents aborde aux îles « hautes » du Pacifique. On devine, malgré le pluriel du texte, que l'auteur fait allusion à l'île de Pâques :

[...] Plus loin, plus loin où sont les îles hautes - îles de pierre ponce aux mains de cent tailleurs d'images; lèvres scellées sur le mystère des écritures, pierres levées sur le pourtour des grèves et grandes figures averses aux lippes dédaigneuses... (Vents, IV, 2, 31).

Saint-John Perse combine ici des éléments pris à la lecture d'un ouvrage qui vient de paraître au moment où il compose Vents : L'île de Pâques, d'Alfred Métraux ${ }^{50}$ :

Les artisans formaient des corporations fermées, assez semblables à celles de notre moyen âge. [...]. La seule corporation dont l'existence soit attestée est celle des sculpteurs d'images, qui formaient un groupe privilégié et respecté. Il y a cinquante ans, quelques indigènes parlaient avec orgueil de leurs ancêtres qui avaient taillé les grandes statues du volcan (p. 85).

Chapitre intitulé «le mystère des tablettes »: «La civilisation de l'île de Pâques, qui a produit des statues gigantesques et des sanctuaires imposants, a-t-elle aussi connu une écriture hiéroglyphique dont le déchiffrement nous révélerait les mystères de son passé ? [...] » (p. 165-179).

Une simple promenade autour de l'île montre que de toute évidence les mausolées ont été dressés le long du rivage (p. 27).

On a beaucoup parlé aussi de la moue dédaigneuse de ces grands visages. Cette expression est frappante sur les sculptures du volcan dont les lèvres fines et saillantes semblent pleines de mépris (p. 141).

Ces bustes à la lippe dédaigneuse étaient-ils des idoles? (p. 148).

E. C. H. - Comment faut-il interpréter ces références intertextuelles que vous avez découvertes? Quelle est votre réaction de critique face aux emprunts?

C.R. - Les emprunts en question témoignent d'un rapport tout à fait particulier qu'entretient Saint-John Perse avec les sciences humaines de son temps et donc, plus largement, avec le réel ; c'est ce rapport qu'il convient d'interroger, tout autant que les particularités d'une technique d'écriture. Refusant un face-à-face narcissique avec la 
feuille blanche, Saint-John Perse nous épargne un lyrisme de complaisance et enrichit considérablement son domaine d'inspiration. Qu'il puisse, comme nous le montrons ici, inscrire dans un même souffle Sumer et les mers du sud en est un témoignage incontestable. Nous sommes particulièrement sensible à cet humanisme qui peut intégrer, dans un même poème, les peuples de tout temps et de tout lieu, sans pour autant perdre de vue sa propre filiation, comme le dit le Discours de Stockholm: "le poète existait dans l'homme des cavernes, il existera dans l'homme des âges atomiques ${ }^{51}$ ». Le monde d'aujourd'hui n'a-t-il pas un impérieux besoin de cette poésie-là?

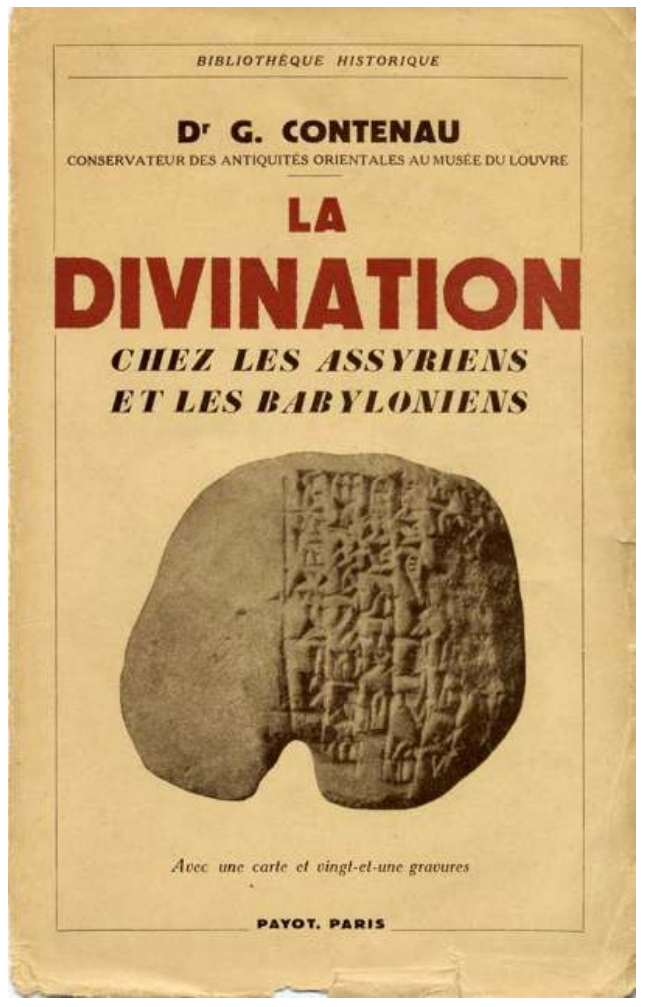






\section{NOTES}

1. Saint-John Perse, Oiseaux, VIII, CEuvres complètes, Paris, Gallimard, coll. «Bibliothèque de la Pléiade ", 1982 [1972], p. 417.

2. Cette découverte a récemment fait l'objet d'un dossier philologique. Voir La Nouvelle Anabase, Revue d'études persiennes, dir. Loïc Céry, n² 2, Paris, L'Harmattan, novembre 2006, p. 141-216.

3. Ces trois états manuscrits sont déposés à la Fondation Saint-John Perse d'Aix-en-Provence, et portent les cotes Ve 1, Ve 2 et Ve 3. L'état Ve 1, écrit à l'encre bleu-noir, comprend les suites I à IV, et comporte de nombreuses corrections sur le texte et en marge. L'état Ve 2 est un manuscrit d'auteur, dédicacé « Pour Atlanta et Allan S. », et daté « Hundred Acre Island, Me., 1945 », écrit à l'encre bleu-noir, avec de très rares corrections à l'encre noire sur le texte. L'état Ve 3 consiste en des épreuves corrigées pour l'édition originale, où paraissent de nombreuses corrections d'auteur et des indications typographiques.

4. Voir Christian Rivoire, «"Et des grands livres pénétrés de la pensée du vent, où sont-ils donc? Nous en ferions notre pâture": À la source des vents. Des emprunts de Saint-John Perse à Georges Contenau dans 82 versets de Vents ", La Nouvelle Anabase, Revue d'études persiennes, $\mathrm{n}^{\circ} 2$, op. cit., p. 143-176.

5. Voir à ce sujet les ouvrages de Renée Ventresque, Saint-John Perse dans sa bibliothèque, Paris, Champion, 2007 ; de Catherine Mayaux, Saint-John Perse lecteur-poète, Le lettré du monde occidental, Bern, Peter Lang, 2006 ; et de Colette Camelin, Éclat des contraires. La poétique de Saint-John Perse, Paris, Éditions du CNRS, 1998. 
6. Voir Joëlle Gardes-Tamine, «De la multiplicité des apparences à l'unité de l'être : le collage chez Saint-John Perse », Saint-John Perse ou la stratégie de la seiche, Aix-en-Provence, Publication de l'université de Provence, 1996, p. 93-104 ; Catherine Mayaux, « Emprunts, collage et mise en page du poème dans le chant X d'Anabase", dans Pour Saint-John Perse, dir. P. Pinalie, Paris, L'Harmattan, 1988, p. 169-185.

7. C. G. Toussaint (trad.), Le Dict de Padma, Paris, Leroux, 1933.

8. René Berthelot, La Pensée de l'Asie et l'astrobiologie, Paris, Payot, 1938.

9. On en trouvera la liste dans le recensement effectué par Christian Rivoire (op. cit., p. 144).

10. Pour une description plus détaillée du manuscrit en question, voir Christian Rivoire, op. cit., p. 147 sq.

11. Le résultat concret de cette technique d'écriture sera présenté par Christian Rivoire dans le « Relevé des emprunts », La Nouvelle Anabase, dir. Loïc Céry, n 4, Paris, L'Harmattan (à paraître).

12. Christian Rivoire, op. cit., p. 146.

13. Saint-John Perse, Discours de Stockholm, O.C., p. 444.

14. C'est Saint-John Perse qui souligne dans ses notes.

15. Voir à ce sujet Henriette Levillain, «Aux sources du mot poétique: le dictionnaire analogique ", Pour Saint-John Perse, éd. P. Pinalie, Paris, L'Harmattan, 1988, p.157-168; Esa Christine Hartmann, «Le dictionnaire analogique, un instrument de création », Les Manuscrits de Saint-John Perse. Pour une poétique vivante, Paris, L'Harmattan, 2007, p. 148-162.

16. Il s'agit ici d'un emprunt au texte de G. Contenau, p. 64 : « Ce que nous disons de la possibilité pour les différents prêtres d'empiéter un peu sur les attributions de leurs confrères [...] est exprimé par les syllabaires, ces listes où des termes sumériens sont expliqués par des termes sémitiques, tantôt plusieurs par un seul, tantôt un seul par plusieurs » (le soulignement provient de Saint-John Perse).

17. MS N 59, p. 12, 13, 15. Contenau, p. 193. Voir Vents, I, 2, 12.

18. MS N 59, p. 3, 10 (2 fois), 14, 15. Contenau, p. 270. Voir Vents, I, 2, 7.

19. MS N 59, p. 12, feuillet 2, recto, côté droit.

20. Ibid.

21. Ibid.

22. Les expressions empruntées sont marquées en gras.

23. Les soulignements reproduisent ceux de Saint-John Perse; nous avons marqué en caractères gras les emprunts directs.

24. «Que le Poète se fasse entendre, et qu'il dirige le jugement ! (Vents, III, 4) / «Les sièges sont placés, le chef des devins est assis, il dirige le jugement » (G. Contenau, p. 71).

25. «Ainsi quand l'officiant s'avance pour les cérémonies de l'aube, guidé de marche en marche et assisté de toutes parts contre le doute - la tête glabre et les mains nues, et jusqu'à l'ongle, sans défaut - c'est un très prompt message qu'émet aux premiers feux du jour la feuille aromatique de son être » (Vents, III, 6) / « les anciennes représentations de prêtres, dont la tête est glabre » (G. Contenau, p. 66) ; «le chef des devins est assis, il dirige le jugement; il élève la plante aromatique, il murmure : [...] Je vous offre une petite gazelle dont les yeux sont gris, la face belle, l'ongle sans défaut" (ibid., p. 71).

26. Voir à ce sujet Esa Christine Hartmann, Les Manuscrits de Saint-John Perse, op.cit., «Les constantes de l'écriture naissante ».

27. «Mon œuvre, tout entière de recréation, a toujours évolué hors du lieu et du temps : aussi attentive et mémorable qu'elle soit pour moi dans ses incarnations, elle entend échapper à toute référence historique aussi bien que géographique; aussi vécue qu'elle soit pour moi contre l'abstraction, elle entend échapper à toute incidence personnelle. À cet égard, la deuxième partie de mon œuvre publiée ne tend pas moins que la première aux transpositions, stylisations et créations du plan absolu » (Saint-John Perse, O.C., p. 562 sq). Nous soulignons.

28. Les soulignements reproduisent ceux de Saint-John Perse. 
29. Voir Loïc Céry, op. cit., p. 202.

30. « Jadis, l'esprit du dieu se reflétait dans les foies d'aigles entrouverts» (Vents, I, 2, 7). Voir G. Contenau : "l'esprit du dieu se reflète dans le foie sacrifié comme une image dans un miroir, selon l'expression de Platon, dans un passage du Timée traitant de l'hépatoscopie » (p. 238).

31. « n'ai-je pas vu le Voyageur d'antan chanceler et tituber sur la chaussée [...] » (Vents, II, 4, 12). Voir G. Contenau, citant la Bible: «Mais ils chancellent par le vin; ils sont troublés par la bière; sacrificateurs et prophètes chancellent par la bière, ils sont vaincus par le vin et troublés par la bière; ils chancellent en prophétisant, ils vacillent en rendant la justice. Toutes leurs tables sont pleines de vomissements et d'ordures (Ésaïe, XXVIII, 7-8)» (p. 45).

32. Pour la liste complète et la localisation exacte de tous ces cas, voir Christian Rivoire, op. cit., p. $151-176$

33. Loïc Céry, op. cit., p. 194.

34. Voir à ce sujet notre étude de ce passage dans le chapitre "Critique et censure", Les Manuscrits de Saint-John Perse, op. cit., p. 114 sq.

35. Le cercle rond représente un signe d'annotation de Saint-John Perse, qui marque ainsi les passages importants de ses lectures, convoquant l'imagination créatrice.

36. Le soulignement provient de Saint-John Perse.

37. Pour la référence de l'emprunt, voir Christian Rivoire, op. cit., p. 161.

38. Chronique, IV, O.C., p. 395.

39. Nous avons reproduit ici les soulignements figurant dans les notes de Saint-John Perse. Le texte assyrien est reproduit en italique.

40. Nous soulignons.

41. Saint-John Perse, Discours de Stockholm, O.C., p. 444.

42. «Car si la poésie n'est pas, comme on l'a dit, le "réel absolu", elle en est bien la plus proche convoitise et la plus proche appréhension, à cette limite extrême de complicité où le réel dans le poème semble s'informer lui-même » (Discours de Stockholm, O.C., p. 444). Nous soulignons.

43. G. Contenau, op. cit., p. 48.

44. Ibid., p. 56.

45. Ibid., p. 228.

46. Voir à ce sujet Loïc Céry, op. cit., p. 214.

47. Loïc Céry, op. cit., p. 203.

48. Saint-John Perse, Discours de Stockholm, O.C., p. 444.

49. Ibid.

50. Alfred Métraux, L'T̂le de Pâques, Paris, Gallimard, 1941.

51. Saint-John Perse, Discours de Stockholm (O.C., p. 445).

\section{RÉSUMÉS}

Une découverte surprenante permit d'enrichir le dossier génétique du poème Vents (1946) de Saint-John Perse : les notes documentaires manuscrites, rencontrées par Christian Rivoire dans les archives de la Fondation Saint-John Perse d'Aix-en-Provence, livrent des éclaircissements exégétiques de nombreux passages obscurs de l'œuvre, et conduisent à une réorientation considérable de l'interprétation du poème. Prises à partir d'un livre d'anthropologie antique qui, curieusement, ne figure pas dans la bibliothèque personnelle du poète conservée à la Fondation, 
La Divination chez les Assyriens et les Babyloniens du $\mathrm{D}^{\mathrm{r}}$ Georges Contenau, ces notes se constituent en un avant-texte du poème naissant, où émerge une véritable écriture poétique. L'article est suivi d'un entretien avec Christian Rivoire, exposant les motivations idéologiques, les étapes méthodologiques et les conséquences herméneutiques de sa recherche dans les archives.

A surprising discovery enables us to enrich the genetic dossier of Saint-John Perse's poem Vents (1946). The documentary manuscript notes, found by Christian Rivoire in the Saint-John Perse Foundation at Aix-en-Provence, provide exegetic clarifications of many obscure passages in the work, thus leading to a significant reorientation of the poem's interpretation. These notes are taken from an ancient anthropology book, oddly enough absent from the poet's personal library held at the Foundation: La Divination chez les Assyriens et les Babyloniens by Dr Georges Contenau. They constitute an avant-texte of the nascent poem, where a true poetic writing emerges. This article is followed by an interview with Christian Rivoire which shows the ideological motivations, the methodological stages and the hermeneutic consequences of his research in these archives.

Un hallazgo asombroso permitió enriquecer el dossier genético del poema Vientos (1946) de SaintJohn Perse: las notas documentales manuscritas, descubiertas por Christian Rivoire en los archivos de la Fundación Saint-John Perse de Aix-en-Provence, que han brindado esclarecimientos exegéticos de numerosos pasajes oscuros de la obra, suscitando una reorientación considerable en la interpretación del poema. Tomadas de un libro de antropología antigua que, curiosamente, no figura en la biblioteca personal del poeta guardada en la Fundación, La adivinación en los asirios y babilonios, del Dr. Georges Contenau, estas notas representan un pre-texto del poema naciente, del que emerge una verdadera escritura poética. Complementa este artículo una entrevista a Christian Rivoire acerca de las motivaciones ideológicas, las etapas metodológicas y las consecuencias hermenéuticas de su investigación en los archivos.

Eine überraschende Entdeckung erlaubte es, das textgenetische Dossier des Gedichtes Vents (1946) von Saint-John Perse zu bereichern: Die handschriftlichen dokumentarischen Aufzeichnungen, die Christian Rivoire im Archiv der Fondation Saint-John Perse in Aix-en-Provence fand, liefern exegetische Erkenntnisse bezüglich zahlreicher obskurer Passagen und führen zu einer beträchtlichen Neuorientierung im Hinblick auf die Interpretation des Gedichts. Die Exzerpte aus einem Werk der anthropologischen Altertumsforschung, La Divination chez les Assyriens et les Babyloniens, von Dr. Georges Contenau, das seltsamerweise nicht in der persönlichen Bibliothek des Dichters enthalten ist, die in der Fondation aufbewahrt wird, bilden einen avant-texte des in seiner Entstehung befindlichen Gedichtes, in dem sich eine genuin poetische Schreibweise herausbildet. Dem Artikel folgt ein Gespräch mit Christian Rivoire, in dem er die ideologischen Beweggründe sowie die methodologischen Schritte und hermeneutischen Konsequenzen seiner Arbeit im Archiv darlegt.

Uma descoberta surpreendente permitiu enriquecer o dossier genético do poema Vents (1946) de Saint-John Perse: as notas documentais manuscritas, encontradas por Christian Rivoire nos arquivos da Fundação Saint-John Perse de Aix-en-Provence, fornecem esclarecimentos exegéticos a numerosas passagens obscuras da obra, e reorientam consideravelmente a interpretação do poema. Tomadas a partir de um livro de antropologia antiga que, curiosamente, não figura na biblioteca pessoal do poeta conservada na Fundação, La Divination chez les Assyriens et les Babyloniens do Dr. Georges Contenau, estas notas constituem um ante-texto do poema nascente, onde emerge uma verdadeira escrita poética. $O$ artigo é seguido por uma entrevista com Christian Rivoire, expondo as motivações ideológicas, as etapas metodológicas e as consequências hermenêuticas da sua investigação nos arquivos. 
Una scoperta sorprendente permette di arricchire il dossier genetico del poema Vents (1946) di Saint-John Perse. Le note documentarie manoscritte ritrovate da Christian Rivoire negli archivi della Fondazione Saint-John Perse di Aix-en-Provence offrono chiarimenti esegetici di numerosi passaggi oscuri dell'opera e conducono a rivisitare l'interpretazione del poema. Tratte da un libro di antropologia antica che, curiosamente, non faceva parte della biblioteca personale del poeta conservata alla Fondazione - La Divination chez les Assyriens et les Babyloniens di Georges Contenau -, queste note costituiscono un avantesto del poema nascente dove emerge un'autentica scrittura poetica. L'articolo è seguito da un dialogo con Christian Rivoire, che espone le motivazioni ideologiche, le tappe metodologiche e le conseguenze ermeneutiche della sua ricerca negli archivi.

\section{INDEX}

Mots-clés : génétique, genèse, création (processus de), Saint-John Perse, Vents, poème, intertexte, découverte, archives, Mésopotamie, fac-similé, entretien

\section{AUTEUR}

\section{ESA CHRISTINE HARTMANN}

Esa Christine HARTMANN, agrégée de lettres modernes et docteur ès lettres, est l'auteur du livre Les Manuscrits de Saint-John Perse. Pour une poétique vivante, Paris, L'Harmattan, 2007. Parmi ses articles consacrés à la critique génétique figurent : “"À la recherche d'une variante perdue" : la mémoire à l'œuvre sur les manuscrits de Saint-John Perse », Genesis, n²3, 2004, « "Cette contradiction latente qui règne au cœur de tout poème" : a contradição, principio criador nos manuscritos de Saint-John Perse ", Manuscrítica, $\mathrm{n}^{\circ}$ 13, 2005, « Vers un renouveau philologique : Saint-John Perse et la critique génétique », La Nouvelle Anabase, $\mathrm{n}^{\circ}$ 1, janvier 2006, " "De grandes œuvres, feuille à feuille" : Vents et la naissance mythique de l'écriture », La Nouvelle Anabase, $\mathrm{n}^{\circ} 2$, décembre 2006, «La fabrication du Discours de Stockholm », Souffle de Perse, n 14, décembre 2009. esahartmann[arobase]yahoo.fr 